\title{
Landing-gear noise prediction using high-order finite difference schemes
}

\author{
Wen Liu ${ }^{\mathrm{a}, *}$, Jae Wook Kim ${ }^{\mathrm{a}}$, Xin Zhang ${ }^{\mathrm{a}}$, David Angland ${ }^{\mathrm{a}}$, Bastien Caruelle ${ }^{\mathrm{b}}$ \\ ${ }^{a}$ Faculty of Engineering and the Environment, University of Southampton, Southampton, Hampshire SO17 1BJ, UK \\ ${ }^{\mathrm{b}}$ Acoustic Department EEA, Airbus Operation SAS, Toulouse, France
}

\section{A R T I C L E I N F O}

\section{Article history:}

Received 19 June 2012

Received in revised form

24 January 2013

Accepted 27 January 2013

Handling Editor: J. Astley

Available online 7 March 2013

\begin{abstract}
A B S T R A C T
Aerodynamic noise from a generic two-wheel landing-gear model is predicted by a CFD/FW-H hybrid approach. The unsteady flow-field is computed using a compressible Navier-Stokes solver based on high-order finite difference schemes and a fully structured grid. The calculated time history of the surface pressure data is used in an FW-H solver to predict the far-field noise levels. Both aerodynamic and aeroacoustic results are compared to wind tunnel measurements and are found to be in good agreement. The farfield noise was found to vary with the 6th power of the free-stream velocity. Individual contributions from three components, i.e. wheels, axle and strut of the landing-gear model are also investigated to identify the relative contribution to the total noise by each component. It is found that the wheels are the dominant noise source in general. Strong vortex shedding from the axle is the second major contributor to landing-gear noise. This work is part of Airbus LAnding Gear nOise database for CAA validatiON (LAGOON) program with the general purpose of evaluating current CFD/CAA and experimental techniques for airframe noise prediction.
\end{abstract}

(c) 2013 Elsevier Ltd. All rights reserved.

\section{Introduction}

Airframe noise has been recognized as one of the major sources of aircraft noise in approach and landing conditions, when landing-gear and high-lift devices are deployed. For short range aircraft the noise from high-lift devices is the most significant among the airframe components. However, for long range aircraft, the landing-gear tends to be the dominant contributor to the airframe noise [1]. The flow around the aircraft landing-gear is complicated due to the highly nonstreamlined configurations designed for safety and economic reasons, and therefore the prediction of noise from such flows has been difficult and currently relies on empirical tools [2-5]. These tools, based on Curle's theory [6], decompose the landing-gear into its major components and compute sound intensities according to the components' dimensions and orientation with respect to the observer locations; and at the end sum up the noise intensities from all the components to provide the overall sound level spectrum. This procedure requires a lot of calibrations with existing test data, therefore has a limitation on predicting noise from unconventional gear architectures at the design stage.

Computational simulation for landing-gear noise prediction would be a general method in principle, which could be applied to any novel landing-gear architecture for future design. In the last decade, simulations of landing-gear have evolved from simplified geometries [7] to realistic detailed configurations requiring large computational meshes [8,9].

\footnotetext{
* Corresponding author. Tel.: +44 7825960478 .

E-mail addresses: Mihero@soton.ac.uk, liuwengemini@hotmail.com (W. Liu).
} 
Unstructured grid methodology has been gaining popularity in the CFD community in recent years with the convenience of grid generation [10]. However, the large meshes (tens of millions of points) required by the unstructured methodology and corresponding low-order finite volume flow solver makes it rather expensive to perform aeroacoustic calculations on complex geometries. Alternatively a structured grid methodology combined with high-order finite difference CAA solver is an option for landing-gear simulation, with the advantages of easy control of local grid resolution, and high spatial accuracy for a relatively small number of cells. Generating a multi-block structured grid for realistic landing-gear configuration is, however, a very challenging task even if the geometry is simplified. The present work aims to apply a high-order accurate, low dispersive and dissipative CAA technique [11-13] to the landing-gear noise prediction. This method could be easily implemented for the structured grid, and is expected to provide accurate landing-gear noise predictions using a relatively small number of grid points.

In this paper, the CFD/FW-H hybrid technique based on high-order CAA methods is implemented for a two-wheel generic landing-gear model, and compared against wind-tunnel test data made available by Airbus. Compressible DES is performed by using fourth-order prefactored compact finite difference schemes [14] and buffer-zone non-reflecting boundary conditions [15]. The current simulation involves 1604 blocks of fully structured meshes where many of them have singular block interfaces across which the gradients of mesh surfaces are discontinuous. A characteristics-based block interface treatment [16] is used in order to avoid the discontinuity problems associated with high-order finite difference schemes. The details of the computed flow-field are presented in this paper, in which the mean flow quantities are compared to experimental data. The unsteady surface pressure data are then used as source terms on an integration surface in an FW-H solver to predict the far-field noise levels and directivity patterns. The radiated noise in the far field is also compared to the experimental data. Individual contribution of wheels, axle and strut to the total noise is investigated separately. In addition, the individual noise signals are analyzed in conjunction with the unsteady flow data from the corresponding components, which provides some insights into the physical mechanisms of landing-gear noise generation. The current work is part of Airbus LAnding Gear nOise database for CAA validatiON [17,18] (LAGOON) program with the purpose of evaluating CFD/CAA and experimental techniques for airframe noise prediction.

The organization of the present paper is as follows. In Section 2, the model geometry and grid generation are described. The numerical methods used for the simulations are presented in Section 3. In Section 4, the aerodynamic results are presented including mean flow comparisons and unsteady flow features. In Section 5, the acoustic results are discussed, and the noise sources are investigated. Finally, conclusions are made in Section 6.

\section{Model description and grid generation}

The simulated model is a scaled generic landing-gear, including a main strut, axle and two wheels. The scale approximately corresponds to 1:2.5 of a nose landing-gear for Airbus A320 (wheel diameter of $300 \mathrm{~mm}$ and the main strut length of approximately $690 \mathrm{~mm}$ ). The strut and axle are circular cylinders and the bottom of the strut is a hemi-sphere. The configuration of the current landing-gear model is depicted in Fig. 1.

The flow conditions in the simulation are provided from the aerodynamic measurements performed in the closed-section wind tunnel F2 [17] in Toulouse, France. The acoustic measurements were made in the CEPRA19 [18] wind tunnel in Saclay, France. The free-stream Mach number was 0.23 , the air density was $1.18 \mathrm{~kg} / \mathrm{m}^{3}$ and the static temperature was $293.56 \mathrm{~K}$.

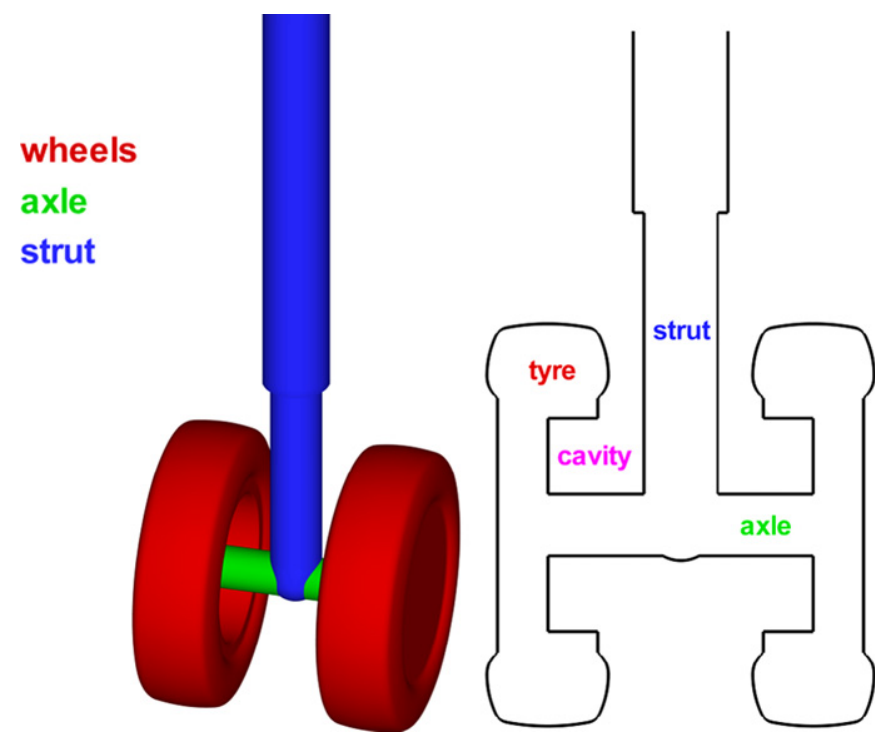

Fig. 1. Two-wheel landing-gear model. 
The entire landing-gear model is surrounded by a multi-block structured grid, which is generated using the commercial software GRIDGEN [19]. Due to the geometrical complexity, a total number of 1604 hexahedral blocks are generated to accommodate the landing-gear model in a domain of $11 D, 6 D$ and $5 D$ in the streamwise, vertical and spanwise direction, respectively, as shown in Fig. 2. The wheel diameter $(D)$ is used as a reference length in this paper. The detailed experimental setup is provided in Refs. [17,18]. It should be mentioned that current simulations are based on the gear setup in the CEPRA19 acoustic measurement, and do not include the wind tunnel floors and support in the F2 aerodynamic measurement. Free-stream velocity is specified on the inflow boundary, and buffer zone boundary conditions [15] are applied in all the other outer boundaries of the domain to remove the reflecting sound waves. No-slip wall boundary conditions are implemented on the landing-gear solid surface.

The wall-surrounding layer consists of 16 grid points normal to the surface to capture the boundary layer, with the first cell width of $1 \times 10^{-4} \mathrm{D}$ in the normal direction and grid stretching ratio of 1.1 . The blocks that enclose the wheel surface have 122 cells distributed around the circumference of the wheel. The baseline grid has approximately 3.5 million cells. A refined grid is also generated to investigate the effect of surface mesh resolution on the acoustic spectrum. The mesh refinement is achieved by doubling the grid points on the wheel circumference and along the strut, but keeping the same resolution in the direction normal to the wall, which results in 15.7 million cells in total. Figs. 3 and 4 show the structured

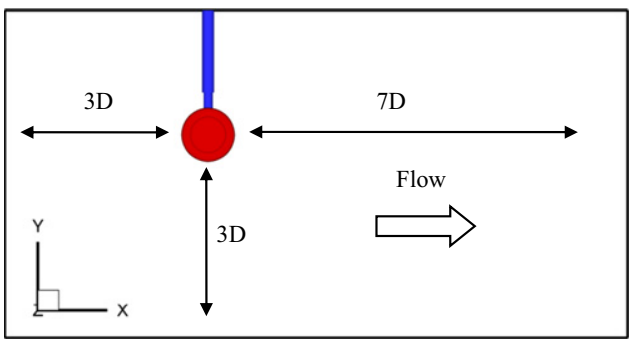

Fig. 2. Schematic of the computational domain.
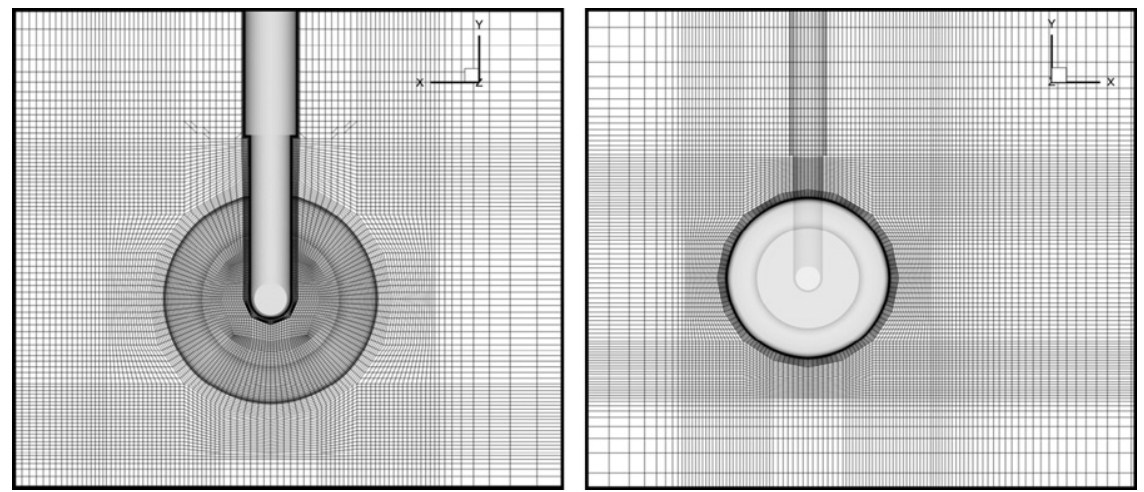

Fig. 3. Structured mesh topology on the $z=0$ plane.
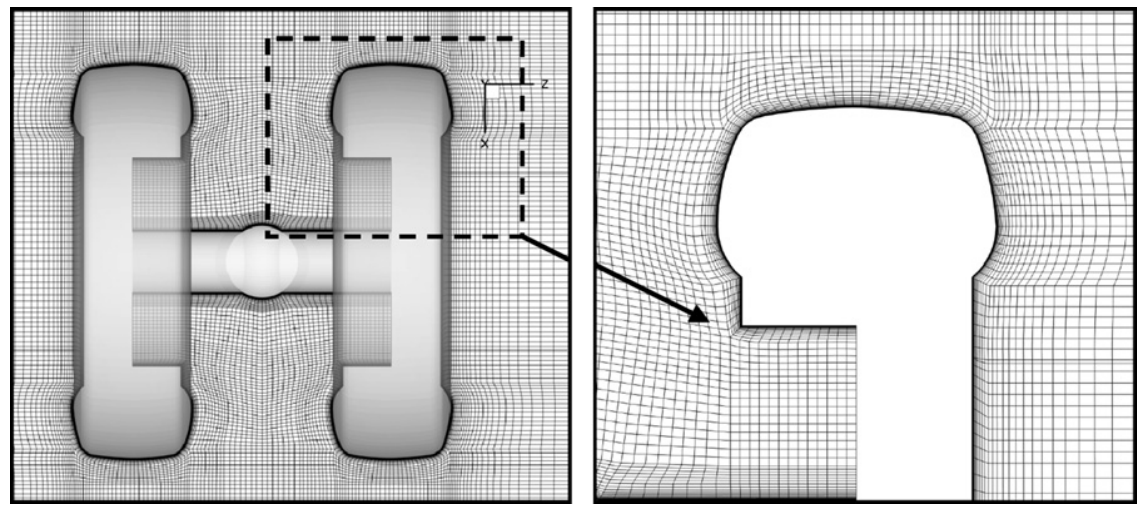

Fig. 4. Structured mesh topology on the $y=0$ plane. 


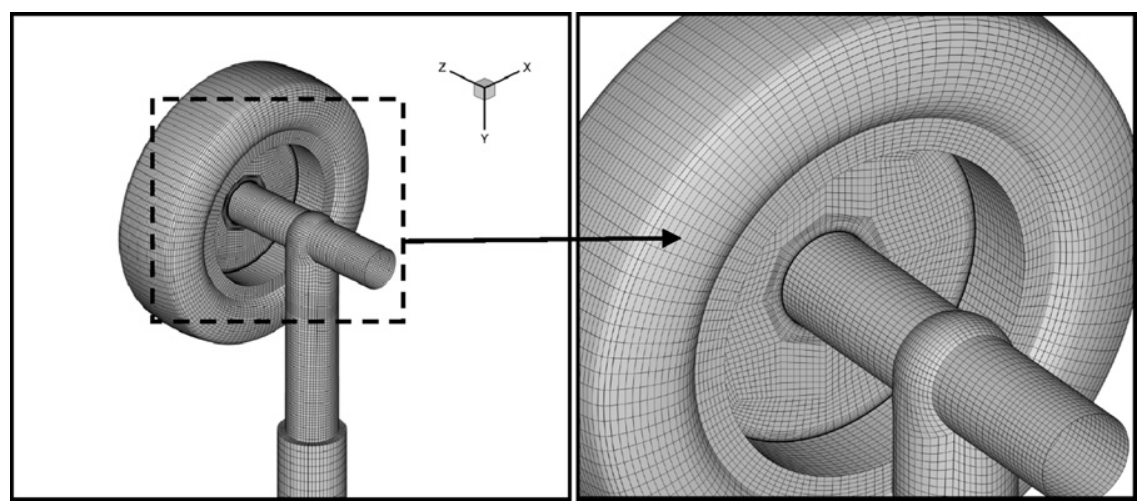

Fig. 5. Surface mesh distributed over the landing-gear walls.

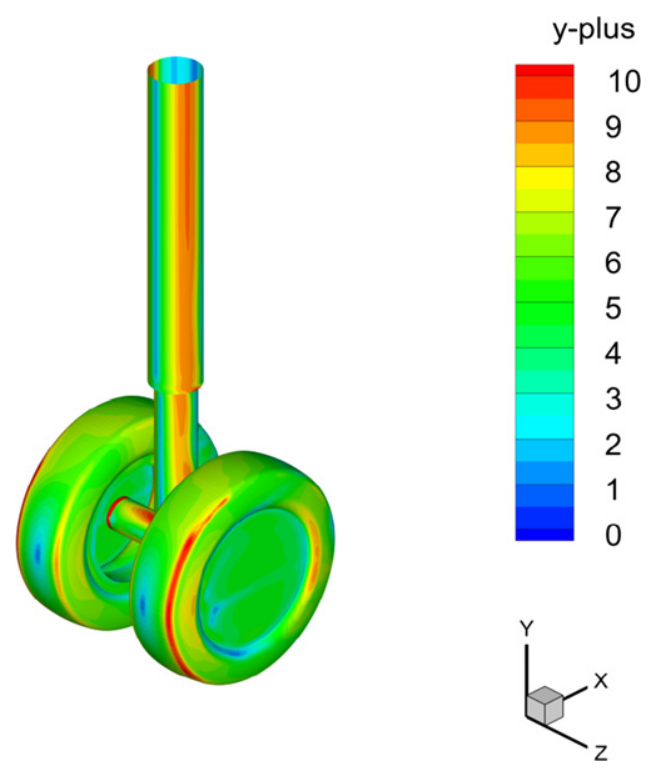

Fig. 6. $y^{+}$Distributions over the landing-gear surface.

mesh topology on the $z=0$ and $y=0$ plane, respectively. The individual structured blocks all have one-to-one connectivity without any overlapping. Fig. 5 shows the surface meshes distributed over the landing-gear walls. Fig. 6 depicts the distribution of $y^{+}$values over the landing-gear surface. The level of difficulties in constructing structured meshes around such a complex geometry (across several hundreds of blocks) prevented the first cell spacing from being kept sufficiently uniform. This resulted in a wider than normal range of surface $y^{+}$values, and therefore an adaptive wall function proposed by Abdol-Hamid et al. [20] was used to compensate the regions with high $y^{+}$values.

\section{Numerical methodology}

The current calculations are performed with an in-house code 'SotonCAA' developed within the Airbus Noise Technology Centre at the University of Southampton. The SotonCAA package includes three parts: (1) a compressible Navier-Stokes solver that calculates near-field unsteady flows; (2) an interpolation code that interpolates the unsteady CFD results onto a user defined FW-H integration surface; (3) an FW-H solver that solves for the noise radiation into far field.

\subsection{Governing equations}

The three-dimensional compressible Navier-Stokes equations in conservative form are solved in generalized coordinates. The current DES strategy is based on the modified one-equation Spalart-Allmaras (SA) turbulence model [21]. The SA length scale, which is normally the distance to the wall, is limited by the grid spacing (cell dimensions). This makes the calculation change smoothly from RANS to LES in regions where the maximum grid spacing of each cell (multiplied by a 
constant of 0.65) is smaller than the distance to the wall [7]. The inflow is fully turbulent, where the initial value of freestream turbulent viscosity ratio (eddy viscosity/laminar viscosity) is 10 .

A FW-H code based on a time-domain integration formulation by Farassat [22] of the FW-H equation is used in the current simulations. It requires the time history of flow variables, such as pressure and velocity components, over a user defined integration surface. Classical velocity scaling laws [6] suggest that dipole surface sources are expected to dominate over quadrupole sources at low Mach number, which seems to suggest a solid integration surface can be used. However, the literature shows widespread difference between the results of solid and permeable integration surfaces in airframe noise simulations. Lockard et al. [23] compared the radiated noise calculated from a solid integration surface and that from permeable integration surface for a simplified landing-gear model. The noise prediction using solid surface sources showed better agreement with the near-field CFD results, and the permeable surface result was suspected to be corrupted by the pseudo-sound created at the closing FW-H surface in the wake. Spalart et al. [24] argued that airframe-noise calculations only using a solid wall integration surface would be inaccurate even at a Mach number as low as 0.115 . They performed DES simulations on a four-wheel rudimentary landing-gear, and compared the far-field noise between solid and permeable integration surfaces. Without the validation of experimental data, the calculated far-field noise from solid surface is consistently weaker than that from permeable surfaces, which may suffer losses of quadrupole sources. Sanders et al. [9] investigated the aerodynamic noise from the same LAGOON landing-gear model described in current paper, using both solid and permeable FW-H surfaces. For the two-wheel landing-gear configuration, the solid surface computation appeared to be globally more accurate than the permeable surface computation, within $2 \mathrm{~dB}$ deviation from the experimental database. The different conclusions from the rudimentary and LAGOON computations may come from the difference in model configurations. The quadrupole sources may be less significant for the two-wheel landing-gear than the four-wheel one where interaction between upstream and downstream components may be significant.

The FW-H surface in this paper is placed on the solid surface only, since the current work focuses on the performance of the high-order finite difference schemes combined with relatively coarse grids (compared to 34 million [9] for the same geometry) in the landing-gear noise simulation. Other uncertainties such as pseudo-sound generated by turbulence crossing the FW-H surface and insufficient grid resolution on the FW-H surface caused by a coarse grid are avoided. Another benefit is that the body-fitted FW-H surface could be used to examine the noise contribution from each individual landing-gear component. Noise contribution is investigated by applying the on-body FW-H surface to axle, strut and wheel separately in an independent manner. This approach does not take into account the effect of interference from the other components that are excluded from the FW-H surface used for one component. Therefore, there exists a certain level of uncertainty in this approach but it may still be used as a reasonable tool to compare noise contribution from different parts of the landing-gear.

\subsection{High-order finite difference schemes}

A fourth-order prefactored compact finite difference scheme [14] is employed for spatial discretization of the governing equations. It is an optimized scheme to provide significantly better resolution than that of standard central differences in the high wavenumber range. The scheme also uses a prefactorization method proposed by Hixon [25] to reduce the non-dissipative central-difference stencil to two low-order biased stencils which have simpler matrices. The advantages of these schemes over traditional compact schemes arise from their reduced stencil size and the independent nature of the factored matrices. It is well known that a major difficulty in dealing with high-order finite difference schemes is the formulation of stable stencils near the boundaries. By reducing the stencil size of the compact scheme the pre-factorization method reduces the required number of boundary stencils and simplifies the boundary specification. In the fourth-order prefactored scheme, the stencil has been reduced from five points to three points (in relation to the original central scheme), and the original tri-diagonal matrix has been replaced by two independent bi-diagonal matrices. In order to close the bi-diagonal matrices, a biased explicit scheme with a 4point stencil is implemented at the domain boundaries; while an 11-point central scheme is employed along the block interface boundaries (without grid singularity) where the flow data are known on both sides.

The high-order schemes are prone to spurious solutions arising from unresolved wavenumbers, particularly in nonlinear calculations on curvilinear grids. Therefore, it is crucial in the current calculations to implement an artificial selective damping model [26] in order to selectively damp or remove the unresolved wavenumber components that would cause spurious solutions. The damping model basically inserts an artificial damping term to the right-hand side of the finite difference equations, and the damping is controlled only in the high wavenumber range by carefully selecting the damping coefficient. In addition, sixth-order explicit filters are applied to the turbulent transport equation to enhance numerical stability by smoothing all the abnormal deleterious perturbations. In SotonCAA, time advancing of solution is performed with an implicit lower-upper approximate factorization algorithm [27]. A pseudo-time technique with subiterations based on Newton-Raphson method is also used to effectively increase the speed of calculations. A dimensionless time step size of 0.001 normalized by the speed of sound and wheel diameter is used, which corresponds to the CFL number of 8.2 with respect to the smallest cell. Five Newton sub-iterations are performed per physical time step.

\subsection{Boundary conditions}

Non-reflecting boundary conditions are crucial to obtain successful results in aeroacoustic calculations since the computational domain is truncated and reflections of waves at the boundaries into the domain are numerical artifacts. In 
the current work, a buffer zone boundary condition [15] is implemented at the outer boundaries to absorb reflected waves. The high-order finite difference scheme in the current simulations requires a fully structured grid, which is highly challenging for such a complex geometry as a landing-gear. A structured grid usually has difficulties in solving finite difference equations at interface surfaces/lines, since the grid matrices are discontinuous along the interface where abrupt changes in the slope of a grid line may occur. Though the discontinuity can be avoided for very simple geometries by generating smooth mesh across multiple blocks, it is extremely difficult to avoid it in the landing-gear geometry. The multi-block characteristic interface treatment [16] is implemented in the numerical schemes to solve this critical problem by communicating the flow variables in the characteristic form, avoiding calculating the derivatives of flow flux and grid matrices across the interfaces.

\subsection{Parallel computing approach}

The current calculations are performed on parallel computing clusters by using domain decomposition and Message Passing Interface (MPI) libraries. Approximately $9 \times 10^{4}$ time steps are required to reach a fully developed flow-field from an initialized condition. The current calculations (on the baseline and the refined grid) are run on two supercomputing clusters, Spitfire and IRIDIS3, available at the University of Southampton. The Spitfire cluster is provided by Microsoft Institute for High Performance Computing which was created at the University of Southampton in 2005. The IRIDIS3 cluster was launched in 2010 and has 8064 processor-cores providing over 72TFlops and up to 32GB of memory per processor-core. The calculations are parallelized over 48 and 256 processors on the Spitfire and the IRIDIS3 cluster, respectively. The baseline calculation were run up to approximately 200 time steps per physical hour on the Spitfire cluster and 1000 time steps per hour on the IRIDIS3 cluster. The refined calculation yields around 250 time steps per physical hour on the IRIDIS3 cluster using 256 processors.

\section{Aerodynamic results}

In this section, the results of mean pressure and velocity are compared with the existing measurement data for the validation of the current calculation. Unsteady surface pressures are validated and discussed afterwards in order to describe the noise generation mechanism.

\subsection{Validation of mean flow data}

Mean pressure distribution on the landing-gear model surface is compared with the measurement data provided by Airbus Operation SAS from the F2 wind tunnel test performed in Ref. [17]. The surface pressure data in the simulation are collected at the same locations as the 64 static pressure taps used in the F2 wind tunnel test as shown in Figs. 7 and 8 . The definition of the azimuthal angle is shown in Fig. 7. The landing-gear model was mounted upside-down in the F2 wind tunnel, and the left and right sides refer to the relative positions when the observer is facing the free-stream flow. The left wheel has 37 pressure taps (Nos. 1-37) around the tire circumference on the mid plane of the wheel, 8 taps (Nos. 40-47) cross-aligned at the wheel top, and 2 taps on the horizontal plane near $0^{\circ}$ (Nos. 38-39). The right wheel has 3 taps on the horizontal plane near $0^{\circ}$ (Nos. 48-50). The axle contains taps in two sections at $\pm 45.5 \mathrm{~mm}$ from the strut. The right section has one tap at $0^{\circ}$ (No. 55). The left section has 4 taps at $0^{\circ}$ (No. 51 ), $90^{\circ}$ (No. 52 ), $180^{\circ}$ (No. 53), and $270^{\circ}$ (No. 54 ). The strut contains taps in one circular section and in one straight line. The circular section is located at $120 \mathrm{~mm}$ below the
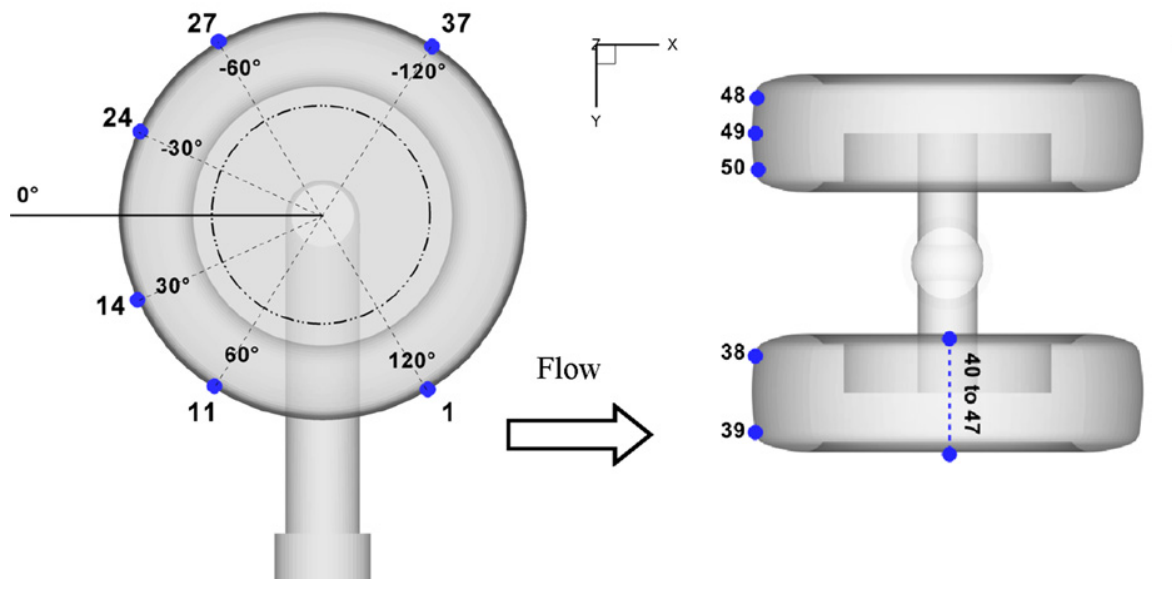

Fig. 7. The location of static pressure taps on the wheels. 


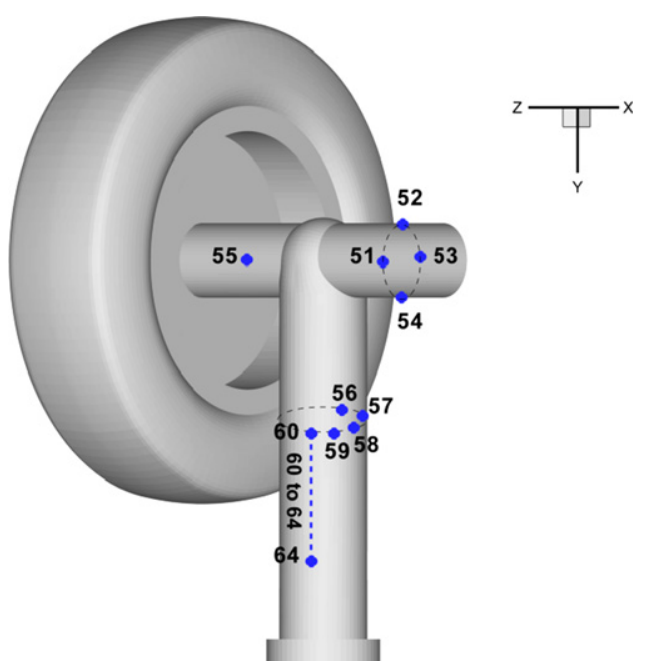

Fig. 8. The location of static pressure taps on the axle and strut.

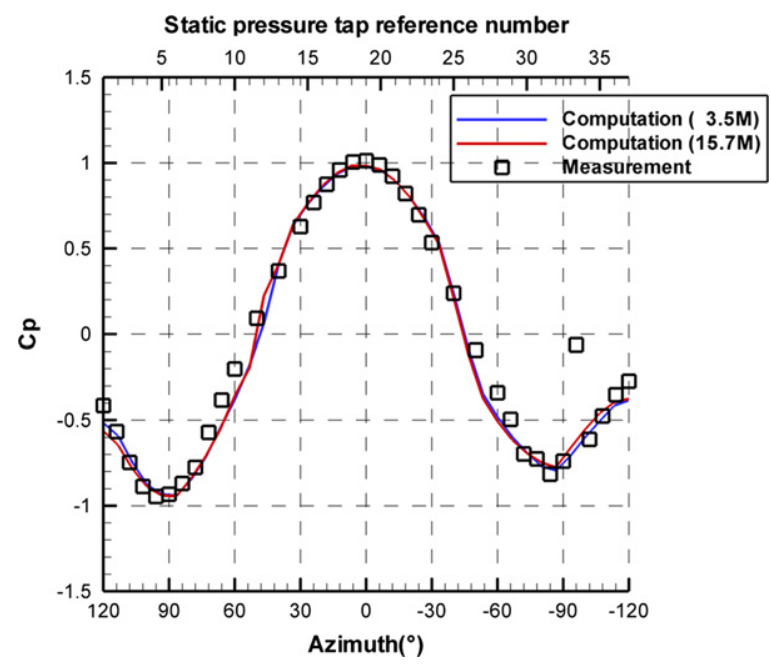

Fig. 9. Mean pressure distribution on the left wheel circumference.

axle, and it has 5 taps at $0^{\circ}$ (No. 60), $-45^{\circ}$ (No. 59), $-90^{\circ}$ (No. 58), $-135^{\circ}$ (No. 57), and $-180^{\circ}$ (No. 56). The tap No. 60 is also the first tap of the straight line of 5 taps (Nos. 60-64) evenly spaced by $20 \mathrm{~mm}$ along the strut at $0^{\circ}$ (stagnation line).

Fig. 9 shows the distribution of mean pressure on the left wheel circumference from $120^{\circ}$ to $-120^{\circ}$ (Nos. $1-37$ ). The pressure levels match very well with the experimental data at most of the locations, except at tap No. 33 where a local discontinuity in pressure occurs in the experimental data. According to Fig. 7, the tap No. 33 is located at an angle of $-96^{\circ}$ on the left wheel, which is close to the top-side of the left wheel. Since there exists no geometric discontinuity around that location, it is speculated that the discontinuity in mean pressure might be attributed to an artifact in the measurement. The two meshes investigated provide similar mean pressure distributions on the wheel surface.

Fig. 10 shows the distribution of mean pressure on the axle and strut of the landing-gear model (Nos. 38-64). The calculated pressure levels on the wheel surface (Nos. 38-50) match very well with the measurement data. There are some disagreements on the axle and the strut surface, especially at tap No. 58. Referring to Fig. 8, No. 58 is located near a flow separation point. The disagreement may be due to the boundary layer tripping used in the experiment. The boundary layer tripping was implemented along the circular dots shown in Fig. 11 aligned at $\pm 60^{\circ}$ with respect to the free-stream direction. Mesh refinement on the landing-gear surface does not have strong influence on the mean pressure distribution on the wheel, but slightly improves the comparisons on the axle and strut.

For the rest of this section, the downstream flow-field is presented based on the results from the refined grid, since both grids provide very similar mean velocity patterns in the wake region of the landing-gear model. Calculated mean velocities are compared with the existing particle image velocimetry (PIV) measurement data from Ref. [17] on two different horizontal planes in Figs. 12 and 13. The streamwise velocity contours are plotted at the top of the figures and the 


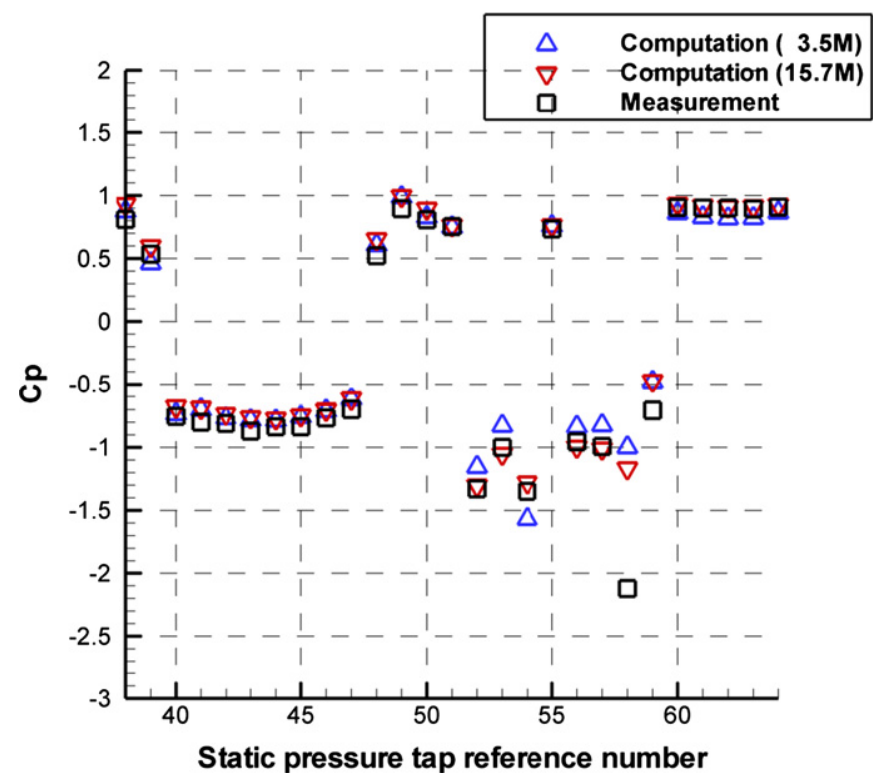

Fig. 10. Mean pressure distribution on the rest of the landing-gear model.

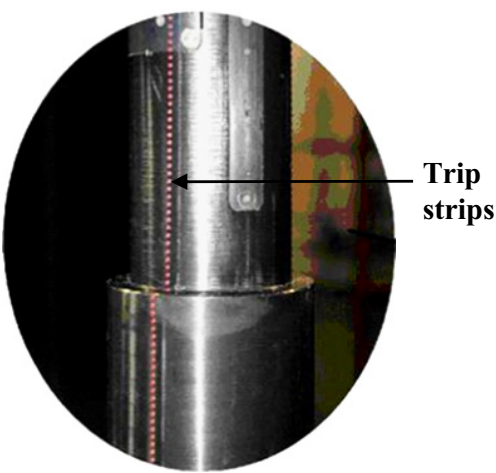

Fig. 11. Boundary layer tripping in the F2 measurement (from Ref. [17]).

spanwise velocity contours are at the bottom. Line plots are extracted at a horizontal plane crosscutting the strut for quantitative comparisons, which are shown in Fig. 14. The velocity contours show a reasonable agreement of the mean velocity field in the downstream wake region of the wheels. It is noticeable that the areas in which separated flows merge behind the wheels are different between the calculation and the measurement. The calculation has a relatively larger merging region with lower streamwise velocity and higher transverse velocity than the measurement does. The discrepancy in the velocity fields in the wake region may be attributed to the following reasons: (1) the current simulations do not include wind tunnel floors and walls in the computational domain in order to perform the acoustic comparison with CEPRA19 open wind tunnel measurement. The wind tunnel effect might be significant on the downstream flow-field. (2) The grid resolution in the direction normal to the wall is relatively coarse, and introduces numerical dissipation due to progressive mesh stretching. The agreement between the calculation and the measurement improves in the strut region as shown in Figs. 13 and 14 as the level of geometric complexity decreases.

\subsection{Unsteady on-surface pressure}

Unsteady surface pressures are compared with the existing experimental data collected by Kulite unsteady pressure sensors. The power spectral density (PSD) comparisons at different unsteady pressure sensor positions (K14 and K24) are depicted in Figs. 15 and 16. K14 is located on the front shoulder of the right wheel, and K24 is located on the strut facing the incoming flow. Good agreement (tonal peaks are well captured) can be found up to $1-2 \mathrm{kHz}$ in the frequency range, above which energy fall-off occurs in the computation. This frequency cut-off is believed to be caused by grid resolution combined with the numerical dissipation. The lowest frequency in the spectrum is $200 \mathrm{~Hz}$, due to the high-pass filter that has been applied to the experimental data. 


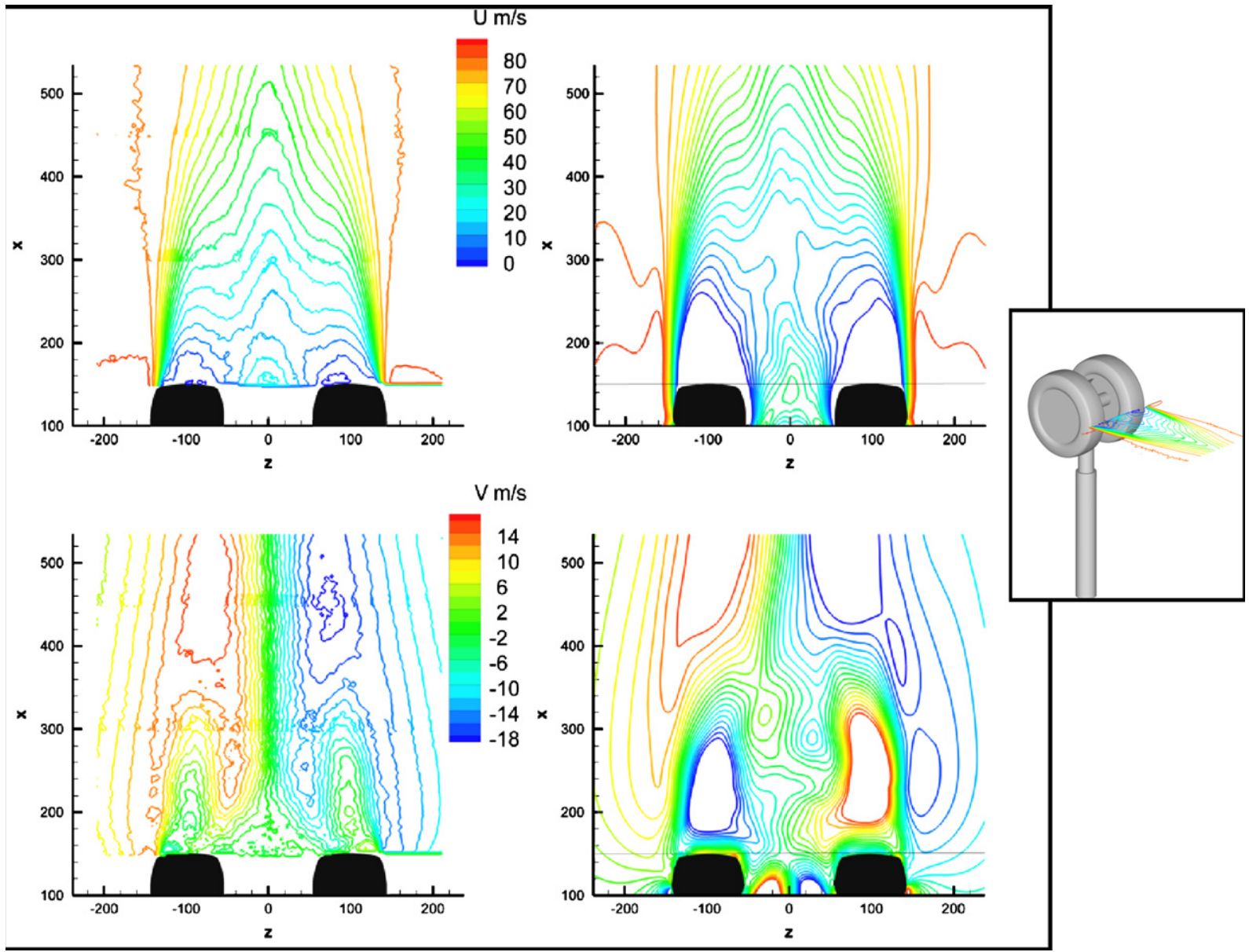

Fig. 12. Mean velocity contour plots on a horizontal plane bisecting the axle. PIV measurement [17] (left) and current calculation (right).

In order to estimate the level of unsteadiness in the flow, the following quantity is defined:

$$
I=\frac{1}{N} \sum_{i=0}^{N}\left(p_{i}-\bar{p}\right)^{2} / p_{\infty}^{2},
$$

where $I$ denotes dimensionless mean square pressure fluctuation, $N$ is the number of samples, $p$ is the instantaneous pressure, $\bar{p}$ represents the mean pressure, and $p_{\infty}$ is the ambient pressure. This measure of pressure fluctuations is useful to identify potentially significant noise source regions particularly close to the body surfaces. Fig. 17 reveals some local spots where high levels of pressure fluctuations dominate. A high level of pressure fluctuations is found on the axle about $\pm 90^{\circ}$ to the freestream velocity vector, which suggests that strong vortex shedding from the axle may potentially be a major contributor to the landing-gear noise. A further investigation on this is detailed in the next section.

\section{Acoustic results}

The FW-H calculations briefed in Section 3.1 are performed using the unsteady surface pressure signals and the predicted far-field radiated sound is discussed in this section. The resulting sound spectra are produced based on 6 blocks of 2048 samples of surface pressure data (50 percent overlapping, 7168 samples in total). Each data group is treated by a Hanning window [28] prior to a Fast Fourier Transform (FFT). The frequency bin width is 24 Hz. The results of the 6 data blocks are then averaged to get the final spectrum.

\subsection{Comparison with experiment}

Power spectral density (PSD) levels are compared between the current computation and the CEPRA19 anechoic wind tunnel measurement in Ref. [18]. Fig. 18 demonstrates the microphone setup in the wind tunnel for the far-field acoustic signal collection. Two microphone arcs are fixed $6 \mathrm{~m}$ away from the model, and 12 microphones are positioned every $10^{\circ}$ 


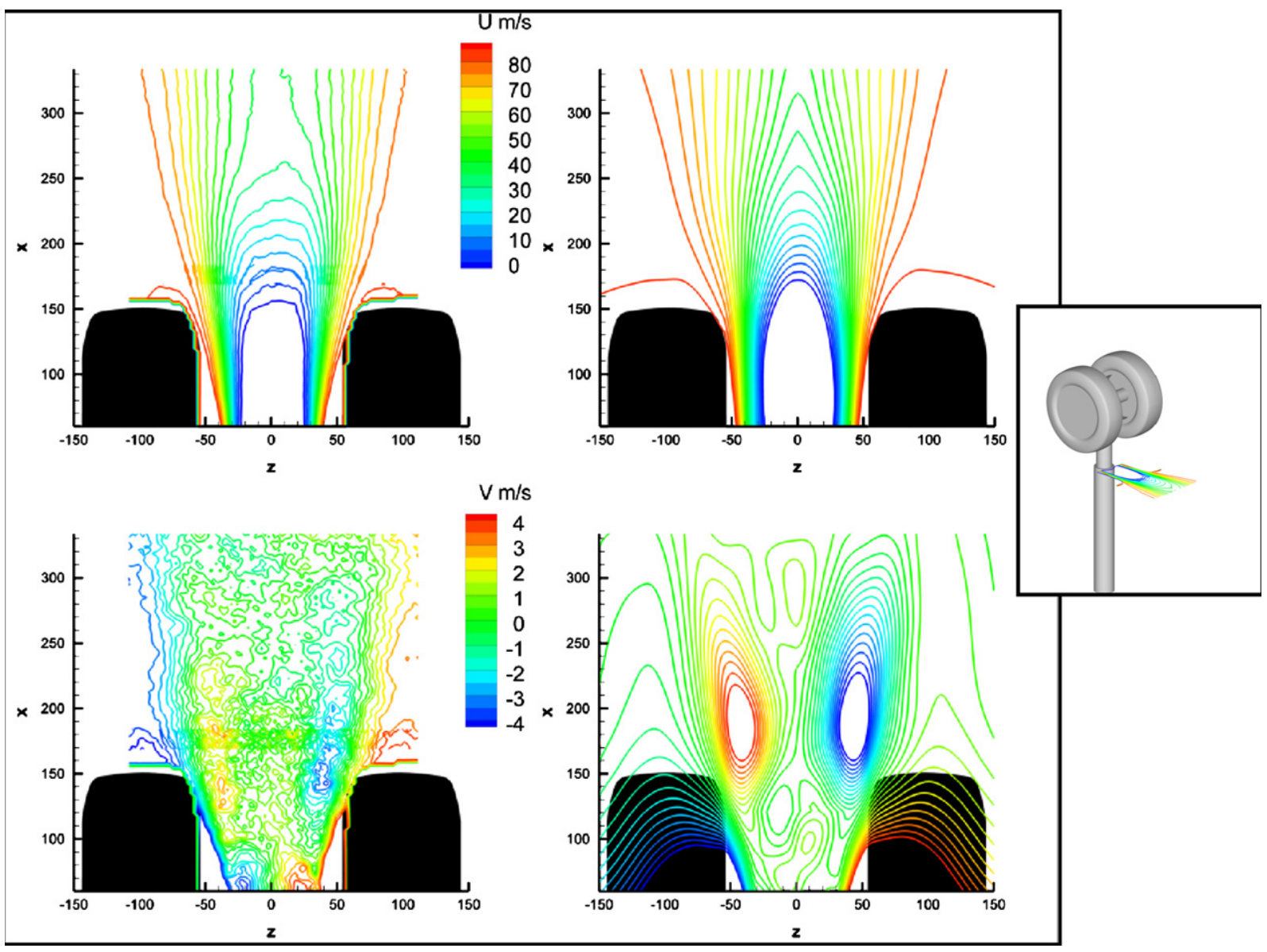

Fig. 13. Mean velocity contour plots on a horizontal plane crosscutting the strut. PIV measurement [17] (left) and current calculation (right).
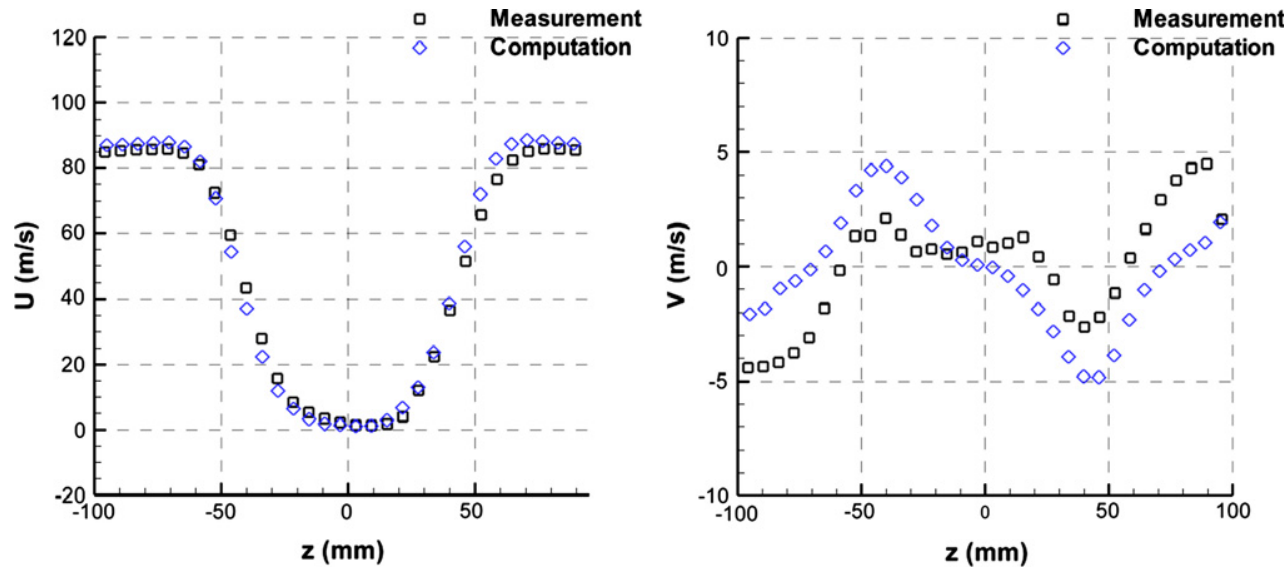

Fig. 14. Mean velocity comparisons on a horizontal plane crosscutting the strut (extracted from $x=160 \mathrm{~mm}$ ).

along each $\operatorname{arc}$ (from $30^{\circ}$ to $140^{\circ}$ in the azimuthal direction). There are two additional microphone arrays and the distance between the microphone arrays and the landing-gear model is $2 \mathrm{~m}$.

For accurate comparison with the current calculation, the experimental data are corrected for background noise, the atmospheric absorption and shear layer refraction effects [29]. Fig. 19 compares the PSD levels between the computation and CEPRA19 measurements on the far-field flyover microphone arc at $140^{\circ}$ in the azimuthal direction. Good agreement is achieved in the frequency range from approximately $200 \mathrm{~Hz}$ to $2 \mathrm{kHz}$ for the baseline grid. The energy fall-off occurring in the mid to high frequency range is caused mainly by numerical dissipation associated with the grid density as well as the 


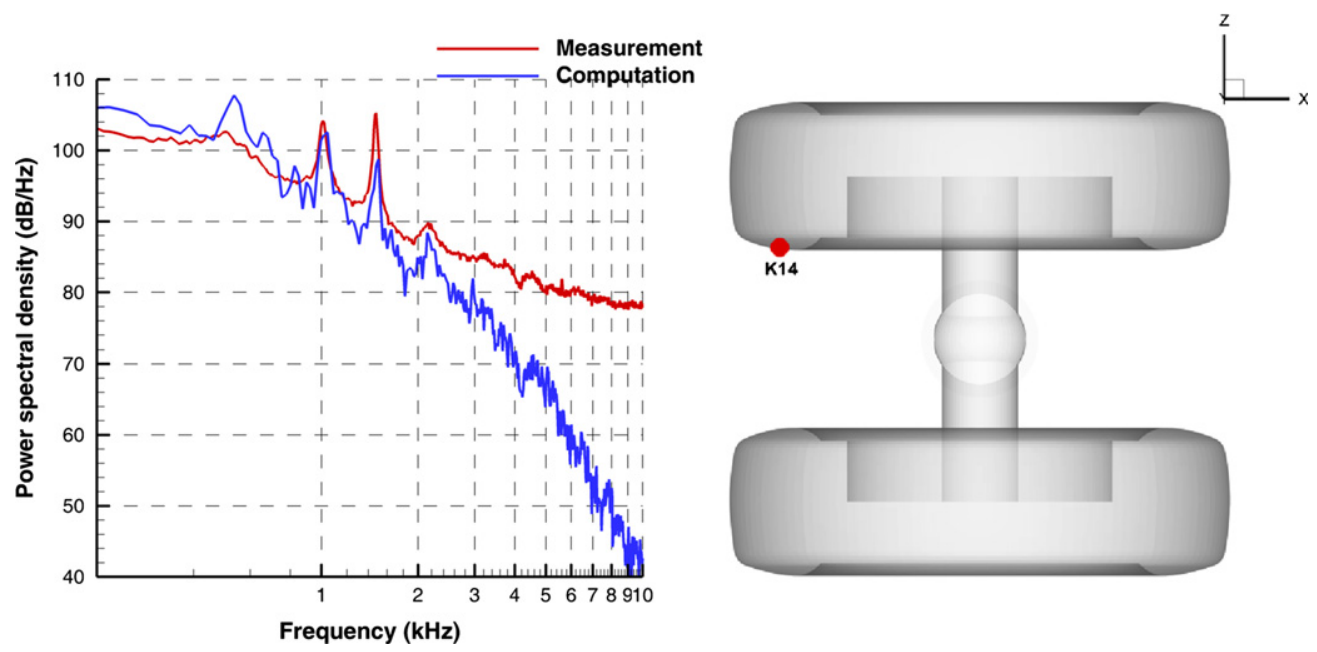

Fig. 15. Surface pressure PSD comparisons at K14.

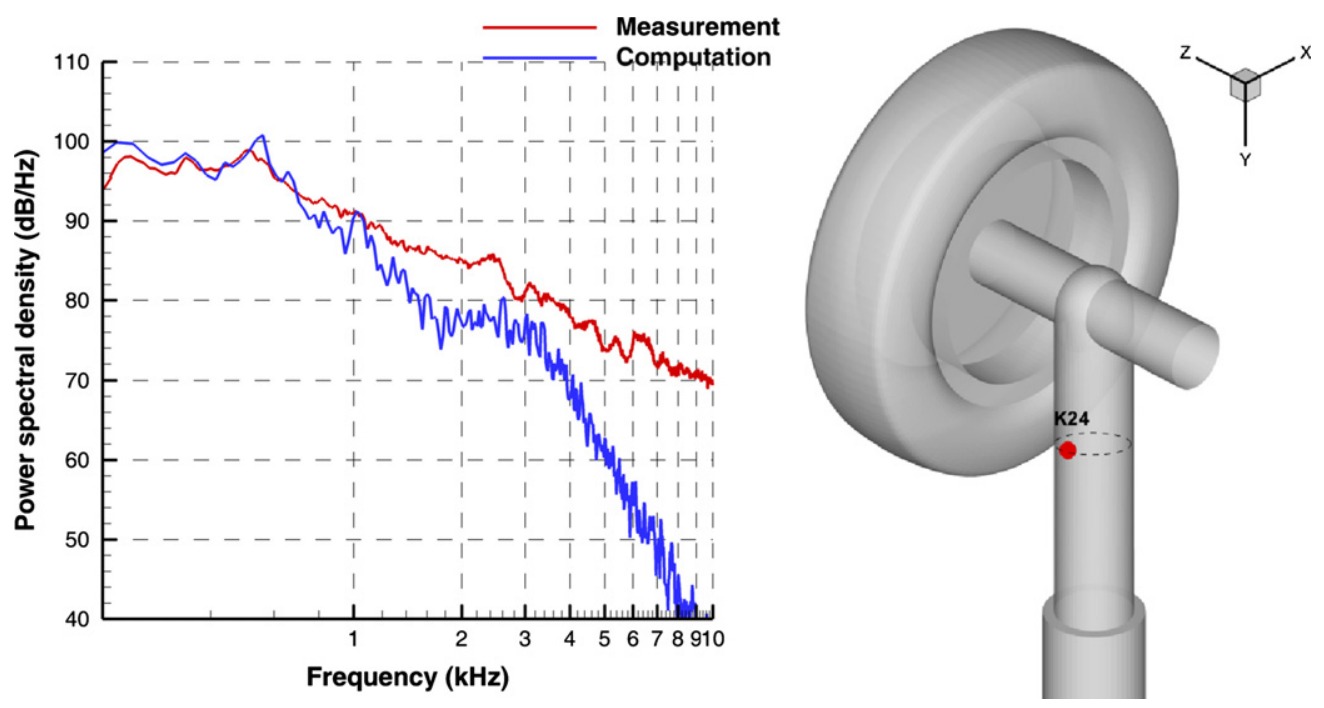

Fig. 16. Surface pressure PSD comparisons at K24.

numerical methods used. Cells of high aspect ratio within the near-wall region are not effective at capturing high wavenumber components.

To investigate the effect of surface mesh resolution on the acoustic spectrum, the grid refinement is made as mentioned in Section 2. Major parameter changes after the grid refinement are shown in Table 1. Fig. 19 shows the improvement in the acoustic prediction by using the refined grid. The fall-off frequency, after using the refined grid, is improved from $2 \mathrm{kHz}$ to $4 \mathrm{kHz}$ approximately.

Fig. 20 shows additional PSD plots from two other microphones: one at the far-field sideline $\operatorname{arc}\left(140^{\circ}\right)$ and the other at the center of the sideline array, respectively. In general, the calculated PSD levels from the refined grid agree with the measurements up to a frequency range of approximately $4 \mathrm{kHz}$. The tonal frequency peaks are also accurately predicted as shown in Fig. 20(b).

The overall sound pressure level (OASPL) is calculated by the integration of the PSD spectrum in the frequency range from $200 \mathrm{~Hz}$ to $25 \mathrm{kHz}$. Fig. 21 shows the comparison of OASPL profiles obtained by the current prediction and the reference measurement across a range of different microphone locations. It can be seen from Fig. 21 that the far-field OASPL prediction is generally in good agreement with the measurement, within a deviation of $2 \mathrm{~dB}$. It is shown that the current methodology and the refined grid used offers accurate acoustic prediction up to a frequency of about $4 \mathrm{kHz}$. It is necessary to investigate the effect of the energy fall-off at $4 \mathrm{kHz}$ in the spectrum on the accuracy of OASPL prediction, i.e. as to whether the energy in the frequency spectrum above $4 \mathrm{kHz}$ contributes significantly to the OASPL. In fact, the OASPL for the landing-gear case is mostly contributed by the low and middle frequency components in the spectrum, and the noise energy contributed by the components above $4 \mathrm{kHz}$ is rather small. This can be verified by performing the 


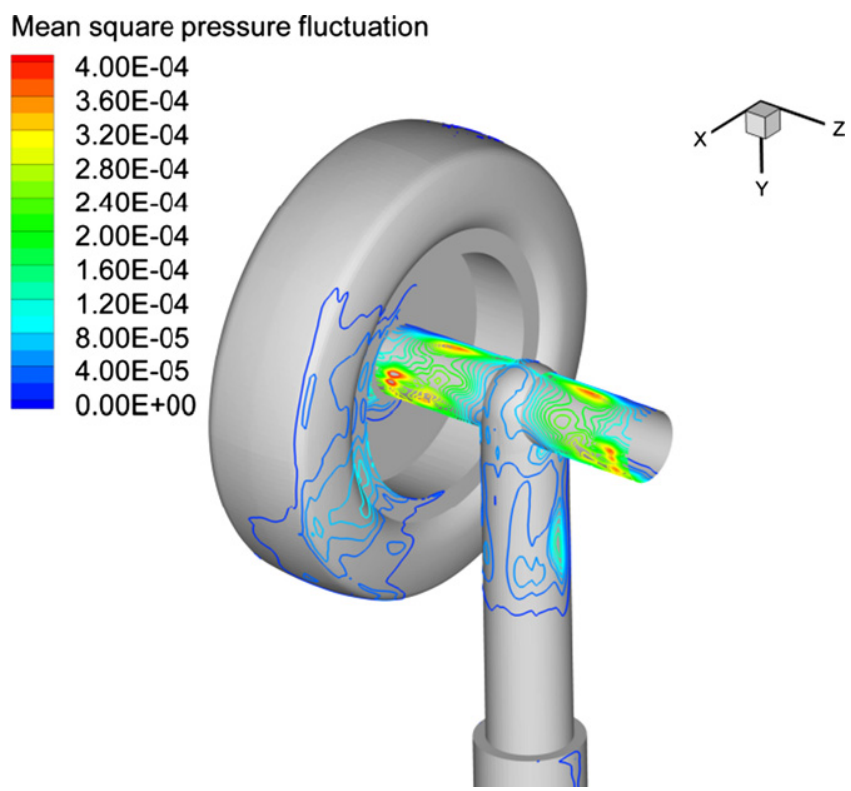

Fig. 17. The mean square pressure fluctuation by Eq. (1) on the landing-gear surfaces.

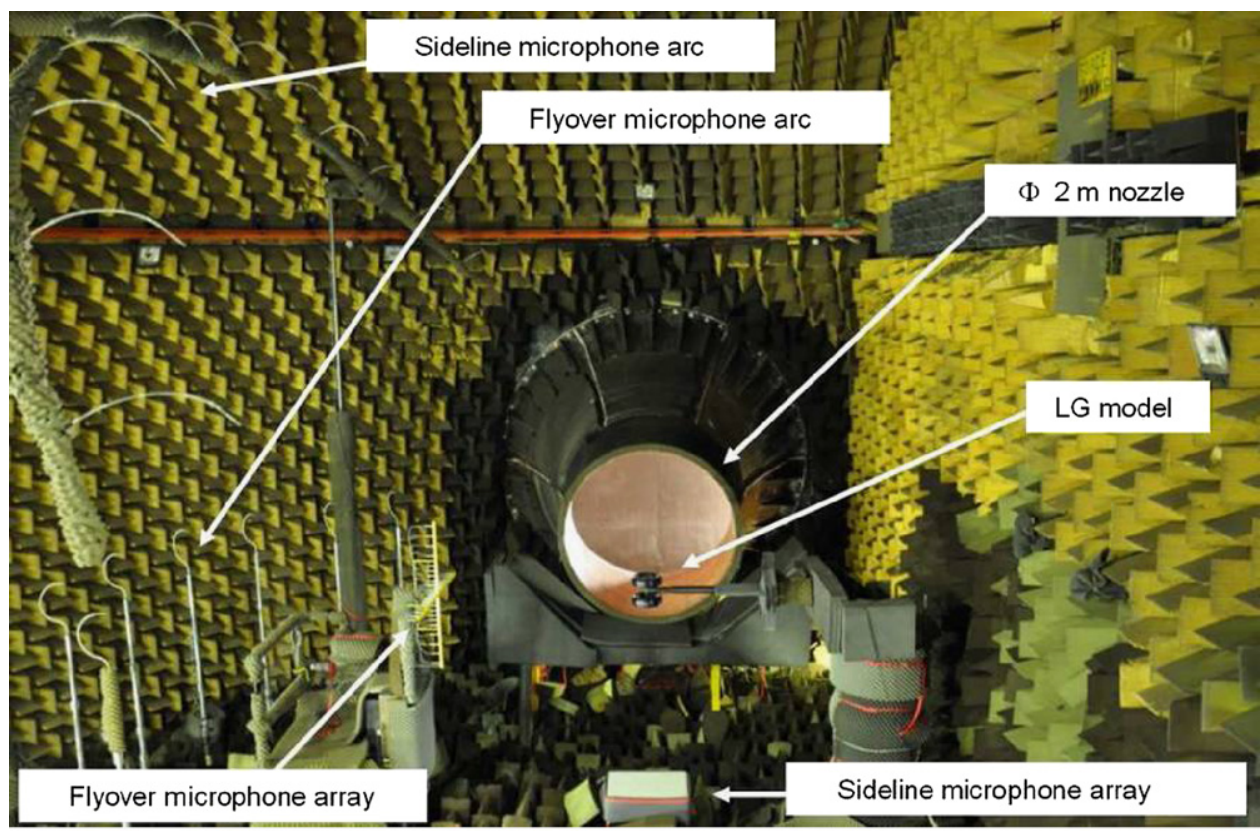

Flyover/Sideline arc

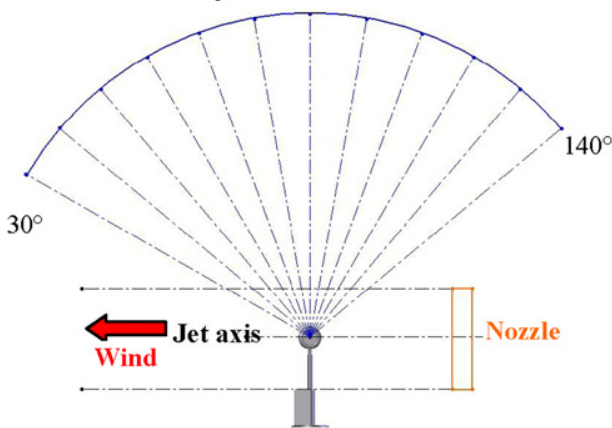

Sideline arc

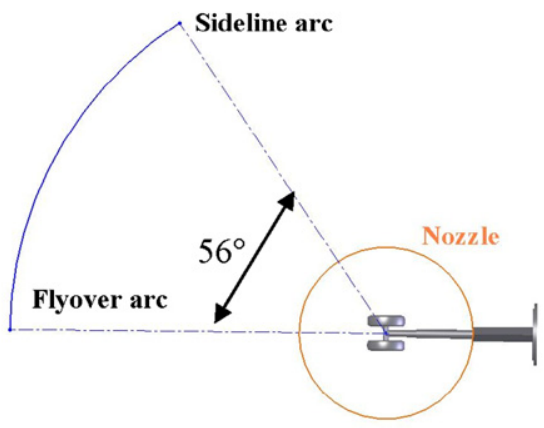

Fig. 18. Microphone setup for the measurement of far-field acoustic signal (from Ref. [18]). 


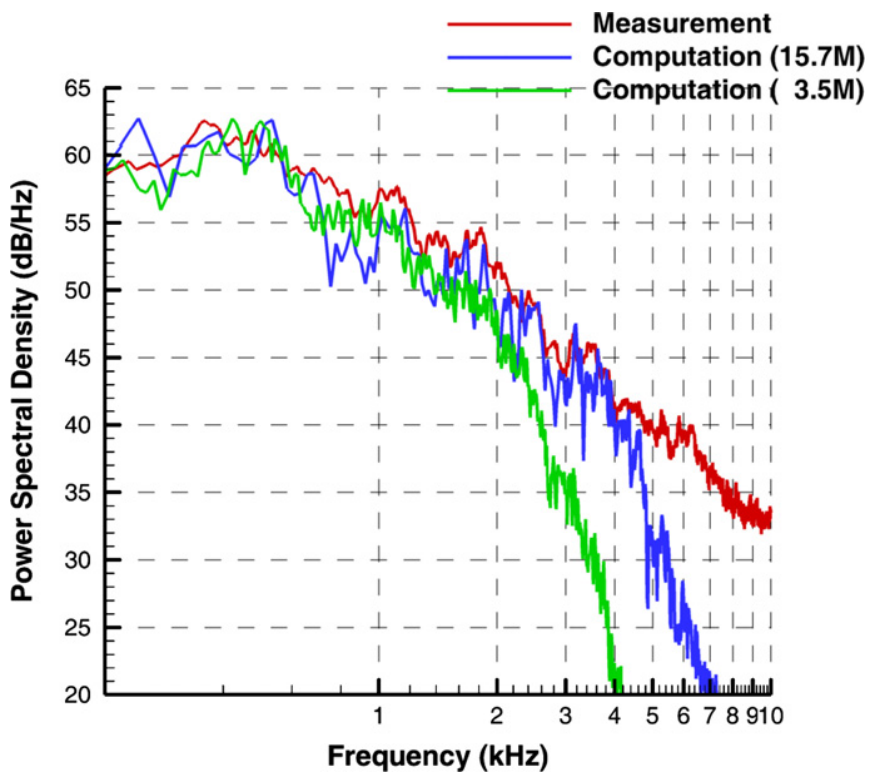

Fig. 19. PSD comparison on the far-field flyover microphone arc at $140^{\circ}$.

Table 1

Comparison between the baseline and the refined grids.

\begin{tabular}{lll}
\hline & Baseline grid & Refined grid \\
\hline Total number of cells & $3.5 \mathrm{M}$ & $15.7 \mathrm{M}$ \\
Number of grid points over the wheel circumference & 122 & 240 \\
Shortest wavelength captured on wheel surface (assuming $10 \mathrm{PPW})(\mathrm{mm})$ & 77.2 & 39.2 \\
Highest resolvable frequency (kHz) & 4.4 \\
\hline
\end{tabular}

(a)

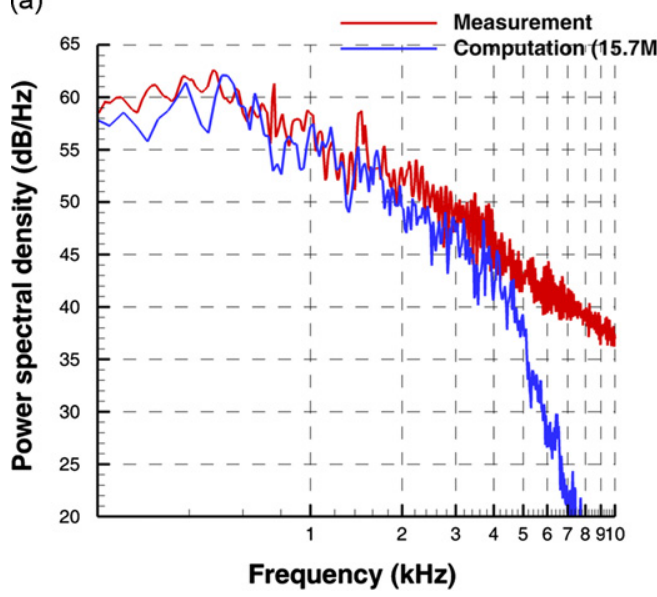

(b)

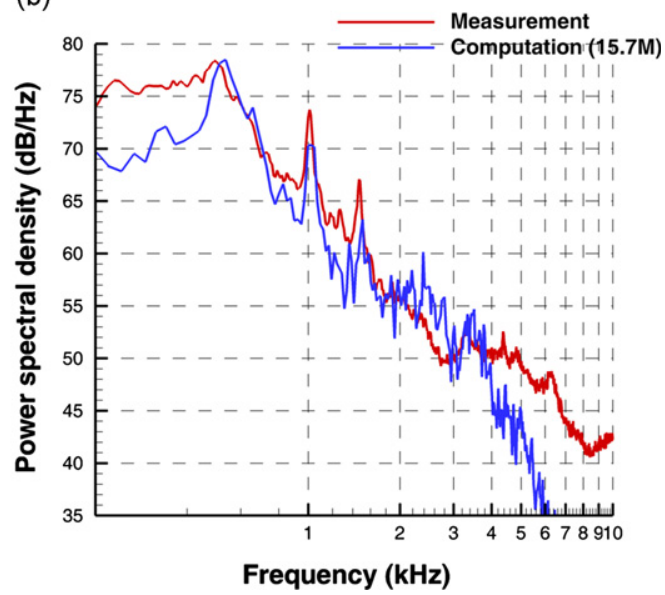

Fig. 20. PSD comparison: (a) on the far-field sideline microphone arc at $140^{\circ}$ and (b) at the center of the sideline microphone array.

integration over the frequencies above $4 \mathrm{kHz}$ in the experimental spectrum, which gives an OASPL value (above $4 \mathrm{kHz}$ ) of less than $0.05 \mathrm{~dB}$. Therefore, the energy loss due to the fall-off at the high frequency would have a limited effect on the overall sound level prediction.

\subsection{Comparison with second-order numerical simulation}

The far-field pressure PSD levels are also compared with the results obtained from a second-order finite volume code, FLUENT, using the same baseline grid of 3.5 million. Fig. 22 shows the PSD at the far-field flyover and the far-field sideline 
(a)

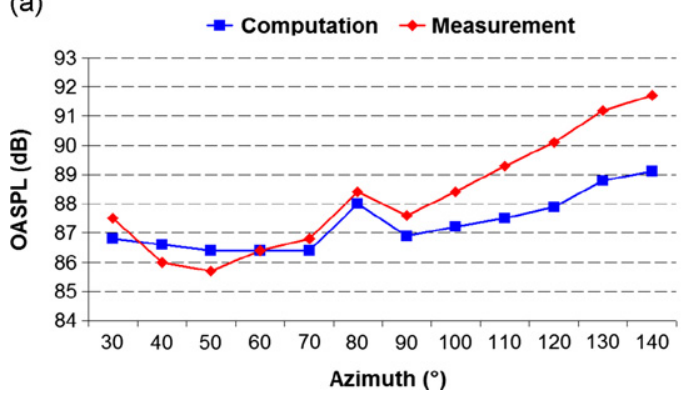

(b)

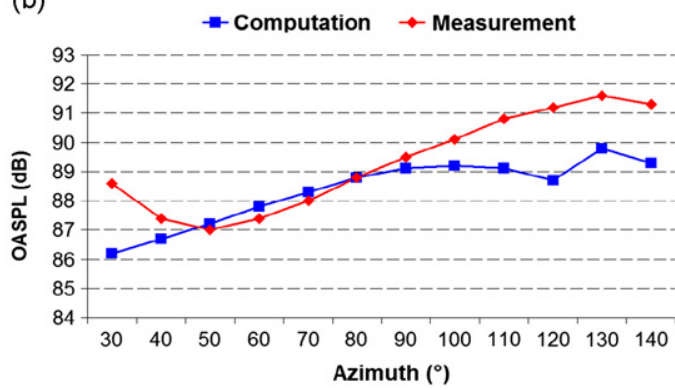

Fig. 21. Far-field OASPL comparison on (a) flyover and (b) sideline microphone arcs.

(a)

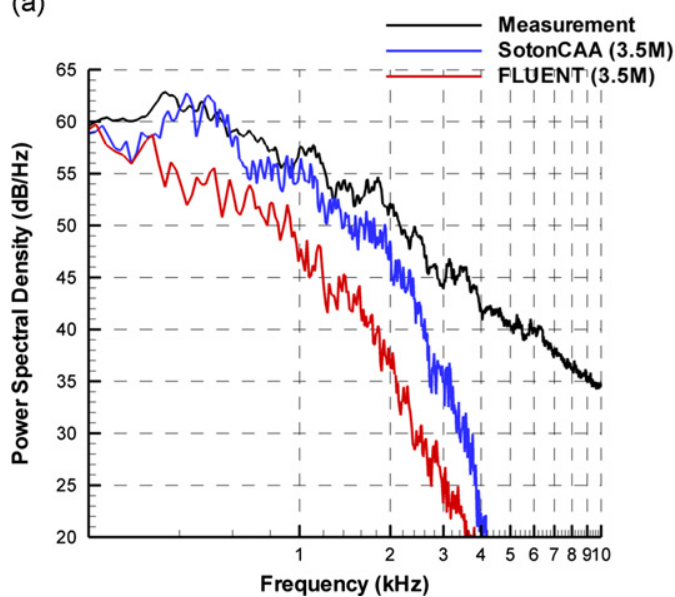

(b)

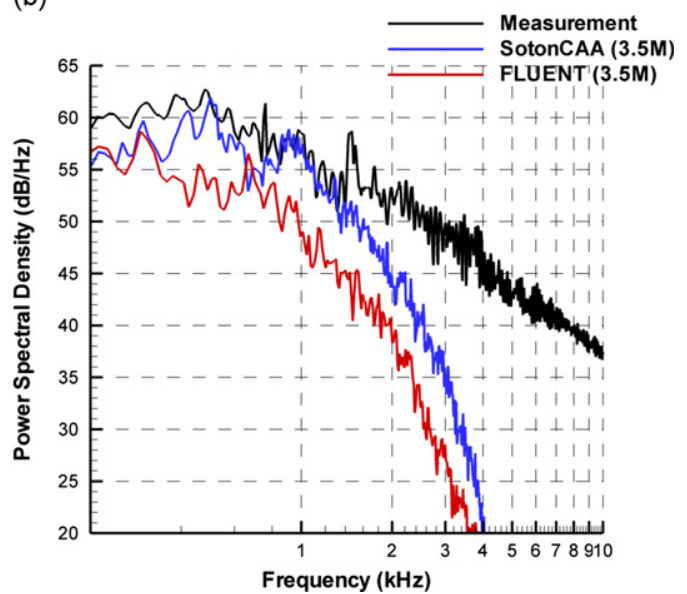

Fig. 22. PSD comparison: (a) on the far-field flyover microphone arc at $140^{\circ}$ and (b) on the far-field sideline microphone arc at $140^{\circ}$.

microphone arcs, respectively. Both sets of data are obtained at an azimuthal angle of $140^{\circ}$ of each arc. The underprediction from the FLUENT simulation is clearly shown in the spectra. This is possibly due to the low-order discretization characteristics of the FLUENT solver since both simulations are carried out on the same computational grid and turbulence model. The comparison suggests that the high-order method presented in this paper is more reliable than the low-order FLUENT solver for the far-field noise computation for a mesh with a relatively small number of cells.

The unsteady near-field and far-field pressures are also compared with the results by Sanders et al. [9], in which the same landing-gear configuration was simulated by the finite volume flow solver elsA using the structured mesh with 34 million grid points. Fig. 23 shows the surface pressure PSD comparison between two computations at K14 on the front shoulder of the right wheel. The far-field pressure PSD comparisons on the flyover and sideline microphone arcs are plotted in Fig. 24.

The predictions from SotonCAA agree well with the results obtained from elsA up to $2 \mathrm{kHz}$ for the surface pressure fluctuations and up to $5 \mathrm{kHz}$ for the far-field acoustic pressures. Sanders et al. showed higher fall-off frequencies than the current computation presumably due to their 34 million grid points compared to the 15.7 million in the current study. Due to the constrictions of one-to-one connectivity and fully structured grids, the cells on the surface are much coarser in this study compared to the grid used by Sanders et al. [9]. In spite of this, the present results show good agreement with the experimental data up to $4 \mathrm{kHz}$ for this landing-gear geometry. Comparing the fall-off frequency of the near-field pressure monitors (in Fig. 23) to that of the far-field observer locations calculated by using an FW-H solver (in Fig. 24), there is a discrepancy between the two fall-off frequencies (also shown in [9]). For the far-field measurements, the fall-off of the numerical simulation compared to the experimental data is above a frequency of $4 \mathrm{kHz}$. However, the on-surface pressures show a fall-off above a frequency of between 1 and $2 \mathrm{kHz}$. It is conjectured that the numerical simulation probably captures the signal components that are correlated over noise radiating areas large enough to contribute to the acoustic integral and does not capture uncorrelated short waves, which then gives better agreement in far-field acoustic spectrum after integration than wall pressure spectrum.

\subsection{Scaling of landing-gear noise with Mach number}

Using the refined grid, a computation at a lower Mach number of 0.18 was performed to explore the scaling of landinggear noise with Mach number. The power spectral density of the pressure at far-field observation positions without 


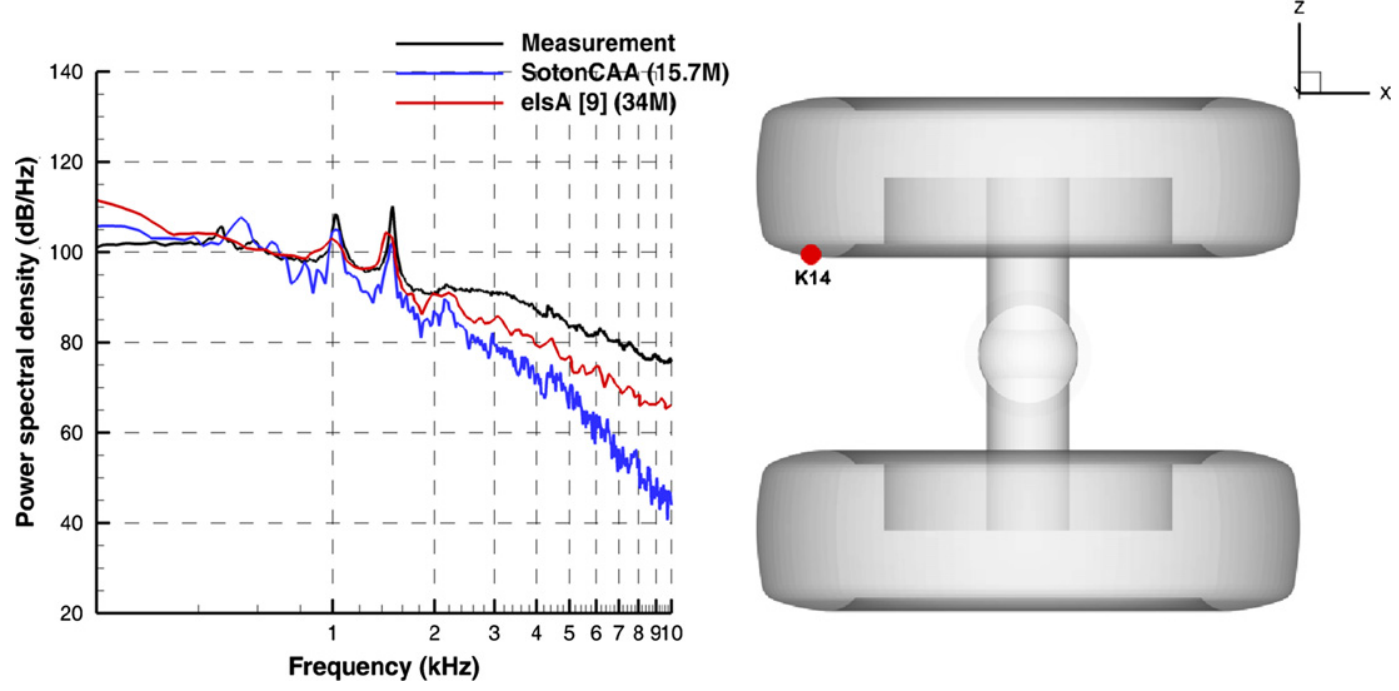

Fig. 23. Surface pressure PSD comparison at K14.

(a)

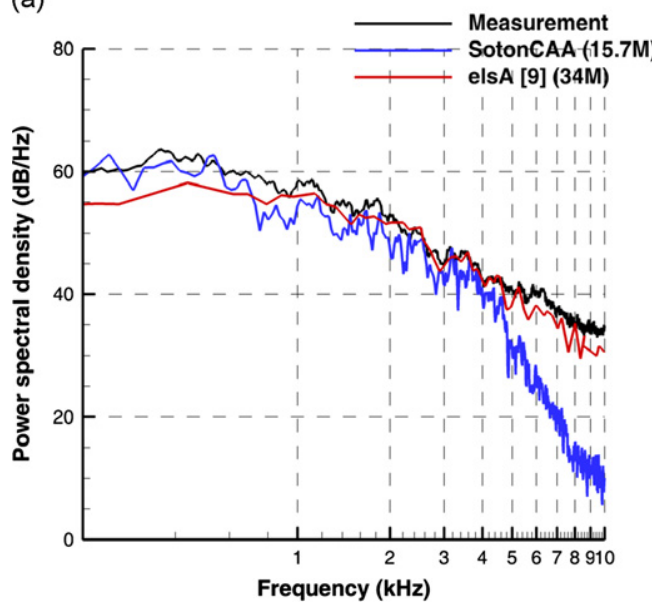

(b)

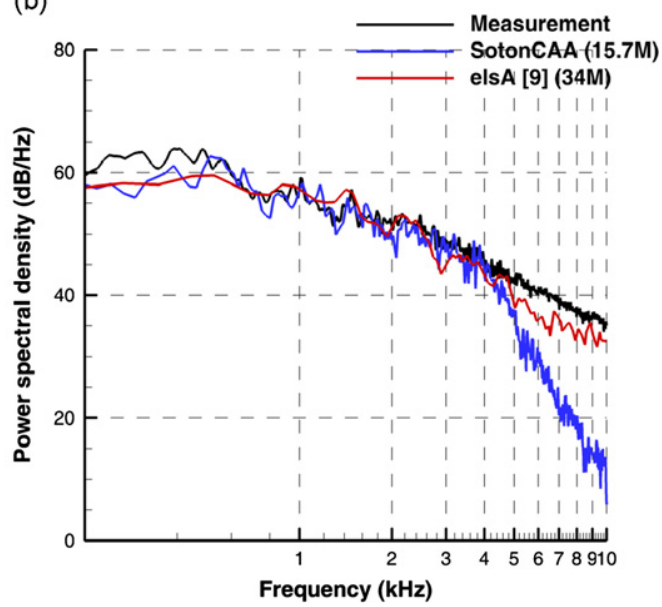

Fig. 24. PSD comparisons: (a) on the far-field flyover microphone arc at $140^{\circ}$ and (b) on the far-field sideline microphone arc at $140^{\circ}$.

amplitude scaling is shown in Fig. 25 to give some perspective on the actual velocity-related differences in the results. The amplitude of PSD has been adjusted assuming either $p^{\prime 2} \propto U^{6}$ or $p^{\prime 2} \propto U^{8}$, shown in Figs. 26 and 27, respectively. The reference velocity that each of the PSD amplitudes with different Mach numbers is adjusted to is $U_{\text {ref }}=0.18$. The power spectral density is plotted against the Strouhal number, St $f \times D / U$, based on the free-stream velocity $U$ and the wheel diameter $D$. Comparing Figs. 26 and 27, the overall shape of the PSD collapses consistently better assuming $p^{\prime 2} \propto U^{6}$ rather than assuming $p^{\prime 2} \propto U^{8}$, especially in the frequency range below $\mathrm{St}=10$ where apparent over scaling occurs assuming $p^{\prime 2} \propto U^{8}$.

\subsection{Noise sources identification}

In this work, noise contribution from each individual gear component is investigated by applying an on-body FW-H surface to axle, strut and wheel separately in an independent manner. Fig. 28 shows the locations at which the far-field OASPL of each component is calculated in flyover and spanwise directions, 20 wheel diameters away from the landing-gear axle center.

Fig. 29 presents the contribution of each individual landing-gear component to OASPL at various azimuthal locations indicated in Fig. 28. The individual contributions are compared to the original OASPL that included all the components. The wheels, which have the largest exposed area to the incoming flow, turn out to be dominant as expected in almost all directions except around $90^{\circ}$ where the axle becomes dominant over the wheels by $2-3 \mathrm{~dB}$. It is believed that the strong axle noise is attributed to the high level of pressure fluctuations on the axle surface that was shown at the end of Section 4 
(a)

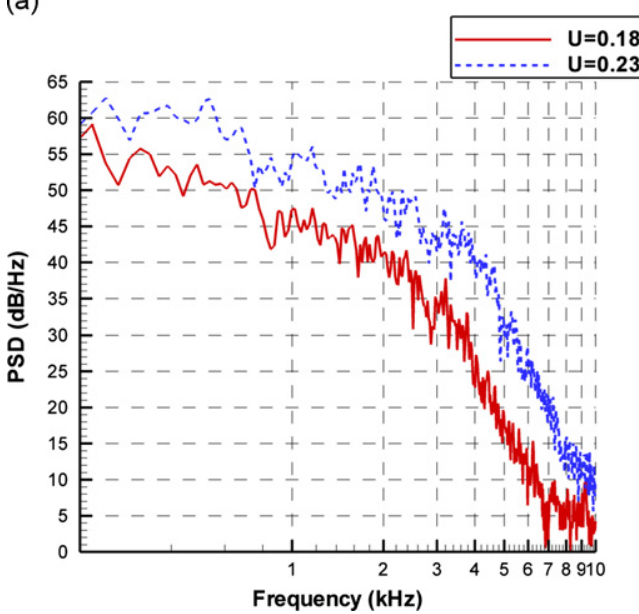

(b)

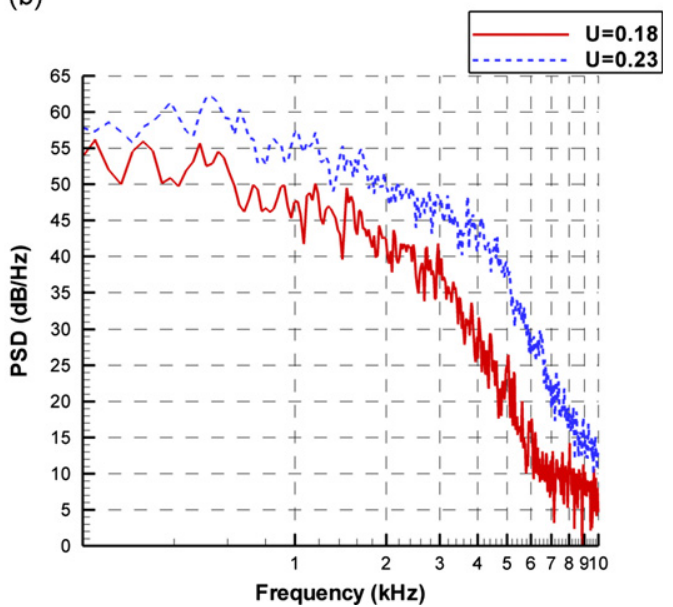

Fig. 25. PSD comparison: (a) on the far-field flyover microphone arc at $140^{\circ}$ and (b) on the far-field sideline microphone arc at $140^{\circ}$.

(a)

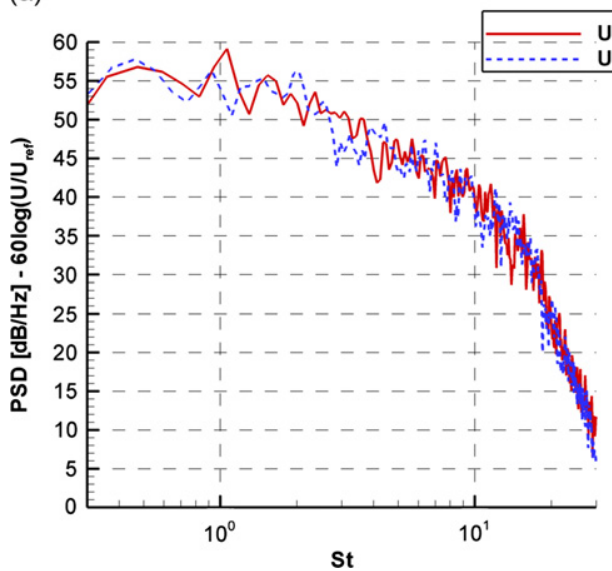

(b)

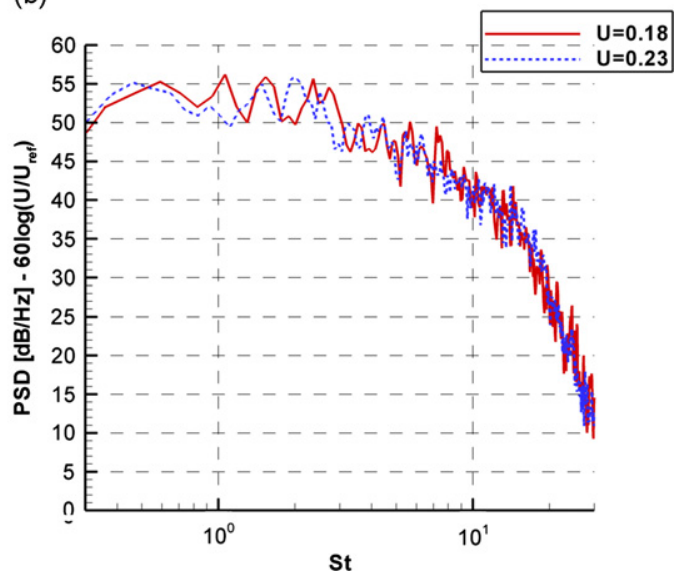

Fig. 26. Scaled PSD comparison: (a) on the far-field flyover microphone arc at $140^{\circ}$ and (b) on the far-field sideline microphone arc at $140^{\circ}$. Scaling performed assuming $p^{\prime 2} \propto U^{6}$ with $U_{\text {ref }}=0.18$.

(a)

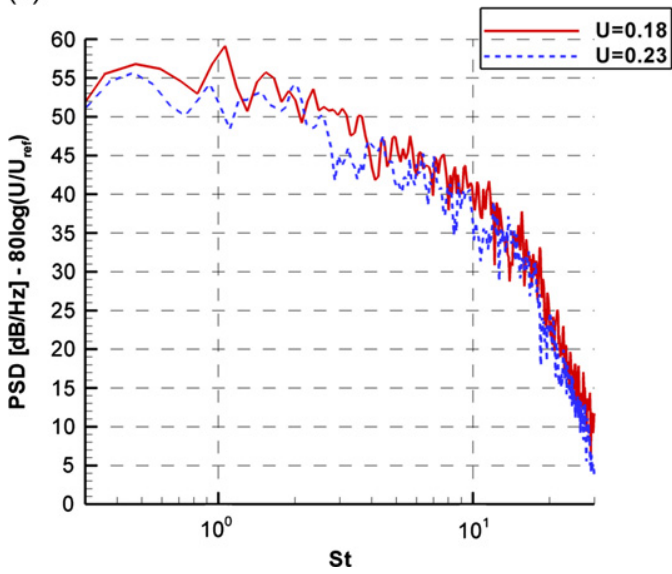

(b)

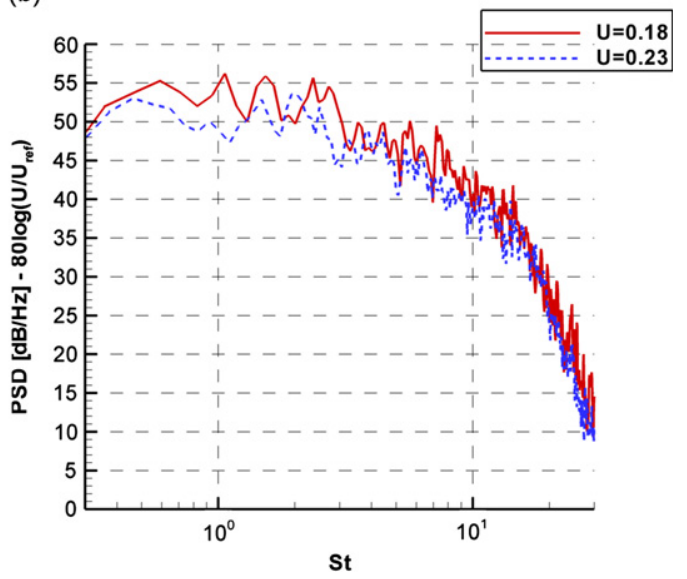

Fig. 27. Scaled PSD comparison: (a) on the far-field flyover microphone arc at $140^{\circ}$ and (b) on the far-field sideline microphone arc at $140^{\circ}$. Scaling performed assuming $p^{\prime 2} \propto U^{8}$ with $U_{\text {ref }}=0.18$. 
(a)

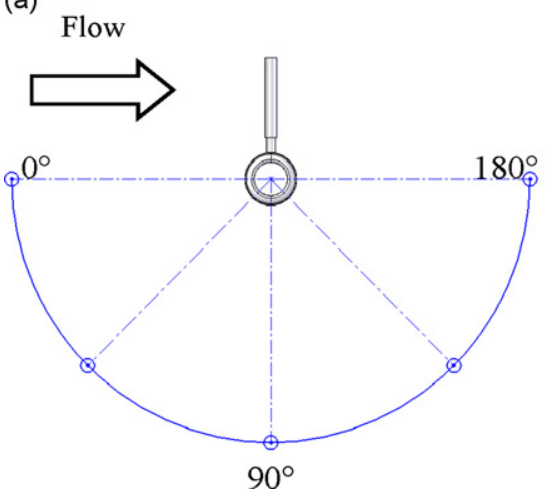

(b)

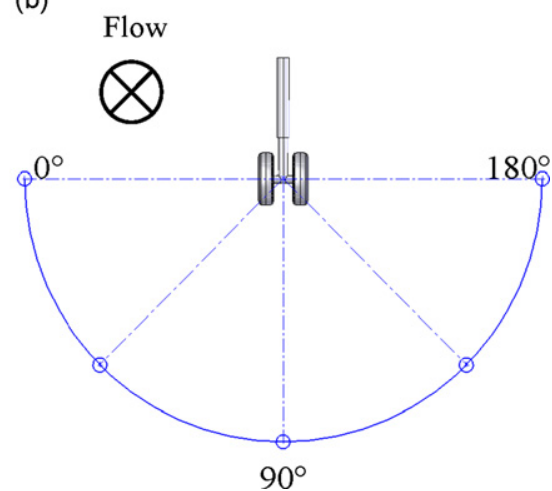

Fig. 28. Far-field observer positions in (a) flyover and (b) spanwise directions.

(a)

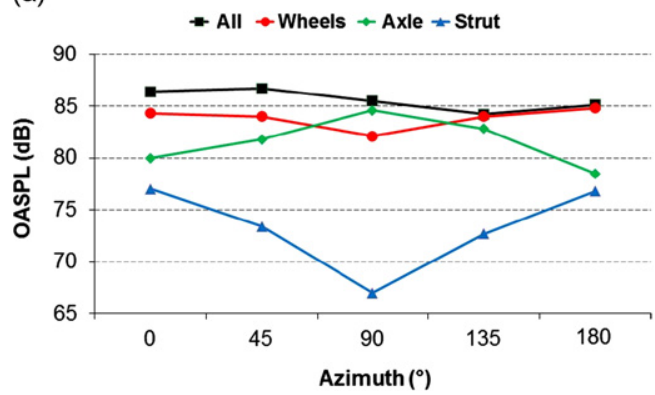

(b)

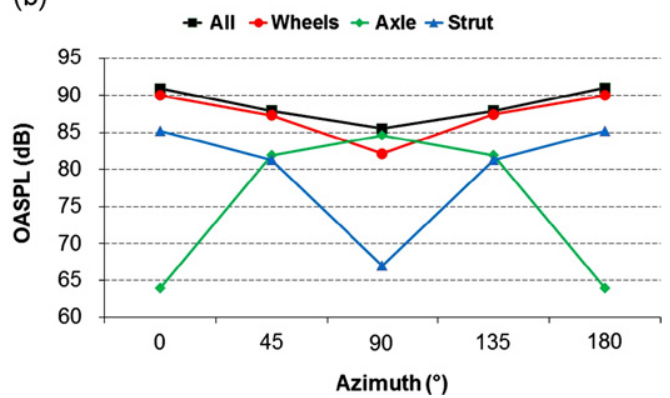

Fig. 29. Noise contribution of each landing-gear component in (a) flyover and (b) spanwise directions.

(Fig. 17). The directivity pattern of the axle noise is consistent with vortex shedding from a circular cylinder. The contribution of the strut is generally weaker than that of the other components; however it radiates relatively strong noise towards $0^{\circ}$ and $180^{\circ}$ in Fig. 29(a) as well as (b) which indicates a typical dipole sound pattern due to its cylindrical shape.

\section{Conclusion}

A high-order computation of landing-gear noise and comparisons with existing experimental data are presented in this paper. It is demonstrated that a multi-block structured grid combined with high-order finite difference schemes and a novel block-interface condition leads to reliable aeroacoustic solutions for such a highly complex geometry with a relatively small number of grid points. Both aerodynamic and acoustic results compare well with the existing wind tunnel measurement data provided by Airbus for the 1:2.5 scaled model of an A320 nose landing-gear at the free-stream Mach number of 0.23 . Far-field noise predictions using a solid FW-H surface showed good agreement with the acoustic measurement for the two-wheel landing-gear configuration. The baseline grid with 3.5 million cells was suitable for mean aerodynamics and produced reasonable acoustic prediction from $200 \mathrm{~Hz}$ to $2 \mathrm{kHz}$. The refined grid with 15.7 million cells increases the highest resolved frequency in the far-field acoustic spectra to $4 \mathrm{kHz}$. The refined grid is shown to be sufficient for accurate prediction of OASPL within $2 \mathrm{~dB}$ of the experimental measurement. The Mach number effect was investigated by collapsing the PSD spectra with those computed in the low Mach simulation at $M=0.18$, and showed that the far-field acoustics scaled roughly with $U^{6}$. The investigation of individual gear components suggests that the wheels emit most of the noise in general but the axle contributes significantly in the direction straight down to the ground (overhead direction from an observer). The axle noise, which is associated with strong vortex shedding from the axle top/bottom surfaces, may be potentially higher than the wheel noise in that particular direction.

\section{Acknowledgments}

This work was supported by Airbus Operation SAS as part of LAnding Gear nOise database and CAA validatiON (LAGOON) project. Supercomputing services are provided by the University of Southampton (IRIDIS3 cluster) and the Microsoft Institute of High Performance Computing (Spitfire cluster). We would also like to acknowledge the members of the Airbus Noise Technology Center (ANTC) at the University of Southampton for their invaluable support. 


\section{References}

[1] W. Dobrzynski, M. Pott-Pollenske, D. Foot, M. Goodwin, Landing gears aerodynamic interaction noise, Proceedings of European Congress on Computational Methods in Applied Sciences and Engineering (ECCOMAS), 2004.

[2] M.G. Smith, L.C. Chow, Prediction Method for Aerodynamic Noise from Aircraft Landing Gear, AIAA Paper 1998-2228, 1998.

[3] M.G. Smith, L.C. Chow, Validation of a Prediction Model for Aerodynamic Noise from Aircraft Landing Gears, AIAA Paper $2002-2581,2002$.

[4] Y.P. Guo, K.J. Yamamoto, R.W. Stocker, An Empirical Model for Landing Gear Noise Prediction, AIAA Paper 2004-2888, 2004.

[5] Y.P. Guo, A Semi-Empirical Model for Aircraft landing Gear Noise Prediction, AIAA Paper 2006-2627, 2006.

[6] N. Curle, The influence of solid boundaries upon aerodynamic sound, Proceedings of the Royal Society of London, Series A: Mathematical and Physical Sciences 231 (1187) (1955) 505-514.

[7] L.S. Hedges, A.K. Travin, P.R. Spalart, Detached-eddy simulations over a simplified landing gear, Journal of Fluid Engineering 124 (2) (2002).

[8] T. Van de Ven, Computational and Aeroacoustic Analysis of A 1/4 Scale G550 Nose Landing Gear and Comparison to NASA \& UFL Wind Tunnel Data, AIAA Paper 2009-3359, 2009.

[9] L. Sanders, E. Manoha, S. Ben Khelil, C. Francois, LAGOON: CFD/CAA Coupling for Landing Gear Noise and Comparison with Experimental Database, AIAA Paper 2011-2822, 2011.

[10] V. Vatsa, D. Lockard, M.R. Khorrami, Application of FUN3D Solver for Aeroacoustics Simulation of a Nose Landing Gear Configuration, AIAA Paper 2011-2820, 2011

[11] C.K.W. Tam, J.C. Webb, Dispersion-relation-preserving finite difference schemes for computational acoustics, Journal of Computational Physics 107 (1993) 262-281.

[12] J.W. Kim, R.D. Sandberg, Efficient parallel computing with a compact finite difference scheme, Computers and Fluids 58 (2012) $70-87$.

[13] S.K. Richards, X.X. Chen, X. Zhang, P.A. Nelson, The evaluation of non-reflecting boundary conditions for duct acoustic computation, Journal of Sound and Vibration 270 (4) (2004) 539-557.

[14] G. Ashcroft, X. Zhang, Optimized prefactored compact schemes, Journal of Computational Physics 190 (2) (2003) $459-477$.

[15] R. Kosloff, D. Kosloff, Absorbing boundaries for wave propagation problems, Journal of Computational Physics 63 (1986) 363-376.

[16] J.W. Kim, D.J. Lee, Characteristic interface conditions for multiblock high-order computation on singular structured grid, AIAA Journal 41 (2003) $2341-2348$.

[17] E. Manoha, J. Bulté, B. Caruelle, Lagoon: An Experimental Database for the Validation of CFD/CAA Methods for Landing Gear Noise Prediction, AIAA Paper 2008-2816, 2008.

[18] E. Manoha, J. Bulté, V. Ciobaca,B. Caruelle, Lagoon: further analysis of aerodynamic experiments and early aeroacoustic results, AIAA Paper 2009-3277, 2009.

[19] Pointwise, Gridgen, URL:〈http://www.pointwise.com/gridgen/〉.

[20] K.S. Abdol-Hamid, B. Lakshmanan, J.R. Carlson, Application of Navier-Stokes Code PAB3D with k- $\varepsilon$ Turbulence Model to Attached and Separated Flows NASA, Technical Paper 3480.

[21] P. Spalart, S. Allmaras, A One Equation Turbulence Model for Aerodynamic Flows, AIAA Paper 92-0439, 1992.

[22] F. Farassat, G.P. Succi, The prediction of helicopter discrete frequency noise, Vertica 7 (1983) 309-320.

[23] D.P. Lockard, M.R. Khorrami,F. Li, High Resolution Calculation of a Simplified Landing Gear, AIAA Paper 2004-2887, 2004.

[24] P.R. Spalart, M. Shur, L., M.K. Strelets, A.K. Travin, Initial noise predictions for rudimentary landing gear, Journal of Sound and Vibration 330 (2011) 4180-4195.

[25] R. Hixon, Prefactored small-stencil compact schemes, Journal of Computational Physics 190 (2) (2000) $459-477$.

[26] J.W. Kim, D.J. Lee, Adaptive nonlinear artificial dissipation model for computational aeroacoustics, AIAA Journal 39 (5) (2001) $810-818$.

[27] J. Li, F. Li, Q. E, A fully implicit method for steady and unsteady viscous flow simulations, International Journal for Numerical Methods in Fluids 43 (2003) 147-163.

[28] A.V. Oppenheim, R.W. Schafer, Discrete-Time Signal Processing, Prentice-Hall, Englewood Cliffs, NJ, USA, 1989, pp. $447-448$.

[29] Onera, Acoustic Tests in CEPRA19 Description of Measurements, LAGOON Deliverable R11, 2010. 